\title{
OBSERVATION OF COHERENT INTERFACIAL OPTICAL PHONONS IN III-V SEMICONDUCTOR NANOSTRUCTURES
}

\author{
Y.-M. Chang ${ }^{a}$, N.-A. Chang ${ }^{a}$, H. H. Lin ${ }^{b}$, C. -T. Chia ${ }^{c}$ and Y. F. Chen ${ }^{d}$ \\ ${ }^{a}$ Center for Condensed Matter Sciences, National Taiwan University \\ ${ }^{b}$ Department of Electrical Engineering, National Taiwan University \\ ${ }^{c}$ Department of Physics, National Taiwan Normal University \\ ${ }^{\mathrm{d}}$ Department of Physics, National Taiwan University \\ No. 1, Sec. 4, Roosevelt Rd., Taipei 10673, Taiwan, R.O.C.
}

Tel: 886-2-33665277 Fax: 886-2-23655404 E-mail: ymchang@)ccms.ntu.edu.tw

\begin{abstract}
We report the coherent phonon spectroscopy of InGaP-GaAs-InGaP SQWs, in which a localized interfacial optical phonon is identified. The GaAs-InGaP heterointerface quality can be characterized by measuring the dephasing time of this interfacial phonon mode.
\end{abstract}

The development of advanced femtosecond laser has made it possible to generate and detect coherent phonons in various condensed matters with optical pump-probe technique [1]. In this paper, the coherent phonon spectroscopy of $7 \mathrm{~nm} \mathrm{GaAs}$-InGaP single quantum wells (SQWs) is performed with time-resolved second-harmonic generation (TRSHG) technique. Spontaneous Raman spectroscopy and time-resolved differential reflectivity (TRDR) measurement are also performed on the same sample for comparison. An interfacial optical phonon at $9.4 \mathrm{THz}$ is identified. This phonon mode is localized at the heterointerface of $\mathrm{GaAs}$ and InGaP. Its dephasing time depends on the sample growth temperature and is governed by the interface quality. The experimental configuration can be found elsewhere [3].

Fig. la shows a typical TRSHG result and its analysis. The oscillatory component is extracted from the slowly varying background and shown in the inset. Its Fourier power spectrum (curve A in Fig. 1b) reveals six coherent optical phonon modes, which are driven via the incident femtosecond laser pump pulses. These six phonon modes are assigned to LO-plasma coupling mode $(8.0 \mathrm{THz})$ of GaAs buffer layer, LO phonon (8.8 THz) of GaAs well [4], interfacial phonon $(9.4 \mathrm{THz})$ of $\mathrm{GaAs} / \mathrm{InGaP}$ interface, LO-plasma coupling mode $(10.0 \mathrm{THz})$, InP-like LO phonon (10.7 THz) and GaP-like LO phonon (11.5 THz) of InGaP barrier [5], respectively. The phonon assignment is confirmed by two other laser spectroscopy techniques: spontaneous Raman scattering (curve B) and time-resolved differential reflectivity measurement (curve C). However, the $9.4 \mathrm{THz}$ peak appeared in TRSHG result (curve A), can only be assigned to an interfacial optical phonon but not any well-characterized bulk phonon mode in either the InGaP barrier nor the GaAs well $[4,5]$. Our experimental results suggest that two driving mechanisms: impulsive stimulated Raman scattering (ISRS) and transient depletion field screening (TDFS) both play major roles in the generation of these coherent phonon modes.

Furthermore, the coherent phonon spectra of three SQW samples are measured, where the samples are grown in the same layer structure but at three different temperature: $520^{\circ} \mathrm{C}, 480^{\circ} \mathrm{C}$, and $440^{\circ} \mathrm{C}$ respectively. It is found that the IP dephasing time decreases from $0.9 \pm 0.2 \mathrm{ps}\left(520^{\circ} \mathrm{C}\right)$ to $0.6 \pm 0.2 \mathrm{ps}\left(440^{\circ} \mathrm{C}\right)$. This result suggests that the higher is the sample growth temperature; the better is the interfacial quality. The dephasing of this IP mode is generally through the following channels: phonon-phonon scattering, phonon-defect scattering and phonon-carrier scattering at the interface region. The oscillatory strength of this IP mode is indeed influenced by the interfacial atomic and electronic structures. According to previous study [6], one general reason for the deterioration of $\mathrm{GaAs} / \mathrm{InGaP}$ interface quality is the formation of low-band-gap temary (InGaAs) or quatemary (InGaAsP) interlayer between the InGaP barrier and GaAs well. Therefore, we tentatively assign the observed IP mode to a local phonon mode confined in this interlayer. R.O.C.

The authors acknowledge the support of National Science Council and Ministry of Education of Taiwan,

\section{References}

[1] T. Dekorsy, G C. Cho, and H. Hurz, in Light Scattering in Solids VIII (Springer, Berlin, 2000), and references therein.

[2] Y.-M. Chang, L. Xu, and H. W. K. Tom, Phys. Rev. B 59, 12220 (1999).

[3] Y.-M. Chang, Appl. Phys. Lett. 80, 2487 (2002).

[4] T. Dekorsy, et. al, Phys. Rev. B 53, 1531 (1996)

[5] G. R. Moriarty, et. al, Thin Solid Films 364, 244 (2000).

[6] Hyeonsik M. Cheong, et. al, Phys. Rev. B 62, 1536 (2000)

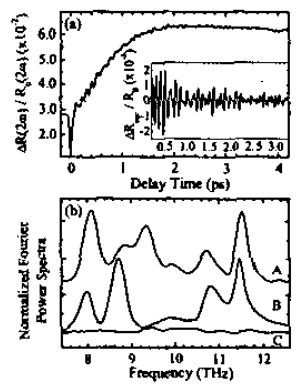

